\title{
Microresonator Defects as Sources of Drifting Cavity Solitons
}

\author{
E. Caboche, F. Pedaci, P. Genevet, S. Barland, M. Giudici, and J. Tredicce \\ Institut Non-linéaire de Nice, UMR 6618 Comité National de la Recherche Scientifique - Université de Nice Sophia-Antipolis, \\ 06560 Valbonne, France \\ G. Tissoni and L. A. Lugiato \\ INFM-CNR and CNISM, Dipartimento di Fisica e Matematica, Università dell'Insubria, Via Valleggio 11, 22100 Como, Italy
} (Received 24 June 2008; published 22 April 2009)

\begin{abstract}
Cavity solitons (CS) are localized structures appearing as single intensity peaks in the homogeneous background of the field emitted by a nonlinear (micro)resonator. In real devices, their position is strongly influenced by the presence of defects in the device structure. In this Letter we show that the interplay between these defects and a phase gradient in the driving field induces the spontaneous formation of a regular sequence of CSs moving in the gradient direction. Hence, defects behave as a device built-in CS source, where the CS generation rate can be set by controlling the system parameters.
\end{abstract}

DOI: 10.1103/PhysRevLett.102.163901

PACS numbers: 42.65.Sf, 05.45.Yv, 42.65.Tg

Localized structures (LS) form in large aspect-ratio media where two or several solutions coexist in the parameter space $[1,2]$. They have been predicted and experimentally observed in many different systems: gas discharges [3], reaction-diffusion systems [4], fluids [5], traveling-wave convection [6], and optics [7-10]. The possibility of exploiting optical localized structures properties for optical memories and information processing has attracted an increasing interest in the last decades [11-13]. These applications are promising when localized structures are created in semiconductor devices, allowing fast response and miniaturization. Single-peak localized structures that appear in semiconductor optical cavities driven by a coherent field (holding beam, HB), also called cavity solitons (CSs), have been theoretically predicted [14] and experimentally observed in broad-area vertical cavity surface emitting lasers (VCSELs) [15]. Recently, several firstdemonstration experiments have been realized to show the potential of these CSs as "pixels" for all-optical processing units $[16,17]$. These functionalities are based on the property of CSs to drift on any parameter gradient with a velocity proportional to the gradient strength [13].

While the existence, the stability and some properties of CSs in semiconductors are nowadays well established, we are still far from understanding their behavior in a "real" device. A major difficulty comes from the engineering of broad-area VCSELs. State-of-the-art fabrication process does not fully prevent from formation of small size (some microns of diameter) "defects" in the transverse plane of the laser. These are in general local spatial variations of the semiconductor resonator characteristics (thickness, electrical and/or optical properties, optical gain) [1820]. Usually, local defects affects detrimentally the control of CS, their positioning [16] and their movement [17,20].

In this Letter we report the experimental and theoretical investigation of a phenomenon never described before: the spontaneous formation of a periodic flow of CSs. This arises from the interplay between a constant gradient in the holding beam phase and hosting material defects which, in this case, play a beneficial role. The frequency and velocity of the flow depend critically on the system parameters, on the external gradient and on the defects characteristics. It is important to point out that the sensitivity to parameter gradients is a general property of LS. For this reason the spatiotemporal behavior presented here is expected to be general to any kind of system supporting localized structures in presence of defects [21]. Similar dynamics were observed in the champagne bubbles, resulting from the interaction between a nucleation site where the bubbles are formed and the buoyancy force [22].

The experimental setup is shown in Fig. 1. A broad-area (200 $\mu \mathrm{m}$ diameter) VCSEL is injected with a collimated and coherent holding beam. Control parameters are detuning between the frequencies of the cavity resonance and of the injected signal $(\theta)$; the intensity of the injected field, and the VCSEL pumping current $J$. These parameters are

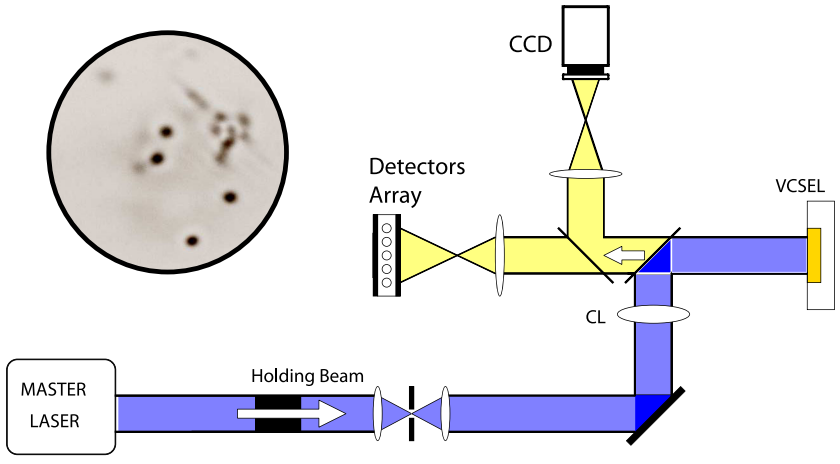

FIG. 1 (color online). Experimental setup. CL: cylindrical lens. ML: tunable master laser. Inset: Transverse profile emission (negative image) of a $200 \mu \mathrm{m}$ section VCSEL, in the regime of CS existence under injection by a broad holding beam. Four CS are present. 
set in the region where CS exist [23]. The inset of Fig. 1 shows the near-field emission profile of the VCSEL with four CS. CS drift is induced by introducing a phase gradient in the holding beam [17]. This is obtained by piezoelectrical tilt of the mirror placed in front of the VCSEL that aligns the holding beam direction with respect to the optical axis of the VCSEL. In order to simplify the experimental analysis, the CS drift is channeled along a line. The holding beam intensity profile is shaped in form of a stripe in the direction of the phase gradient by a cylindrical lens, thus confining the movement of CS within this geometry. Then, monitoring of the CS spatiotemporal dynamics can be obtained by placing an array of four fast $(350 \mathrm{MHz}$ bandwidth) detectors along the channeling stripe in a plane imaging the VCSEL output. A CCD camera allows for near-field time-averaged detection of the VCSEL output.

In Fig. 2, upper panel, we show the time traces corresponding to the VCSEL intensity output picked up by the three detectors $A-C$ placed along the channel line. In particular, detector $A$ monitors a point of the device (that we call point $A$ from now on) where a structure spontaneously appears even if the phase gradient is removed. Each detector records a regular pulsing signal with an average period $\langle T\rangle=63 \mathrm{~ns}$ and the time traces are strongly correlated to each other with a time delay $(\Delta t)$ of $3.0 \mathrm{~ns}$ for two neighbor detectors in the direction of the gradient. For a tiny increase of the VCSEL pumping current $J$ (lower panel), the period of the pulsing signal decreases to $\langle T\rangle=17.5 \mathrm{~ns}$, while the pulse widths and shape are almost unaffected. In left panel of Fig. 3 we plot the average value of period $T$ and its standard deviation as a function of $J$. The decrease of the standard deviation for increasing $J$ indicates an increasing regularity
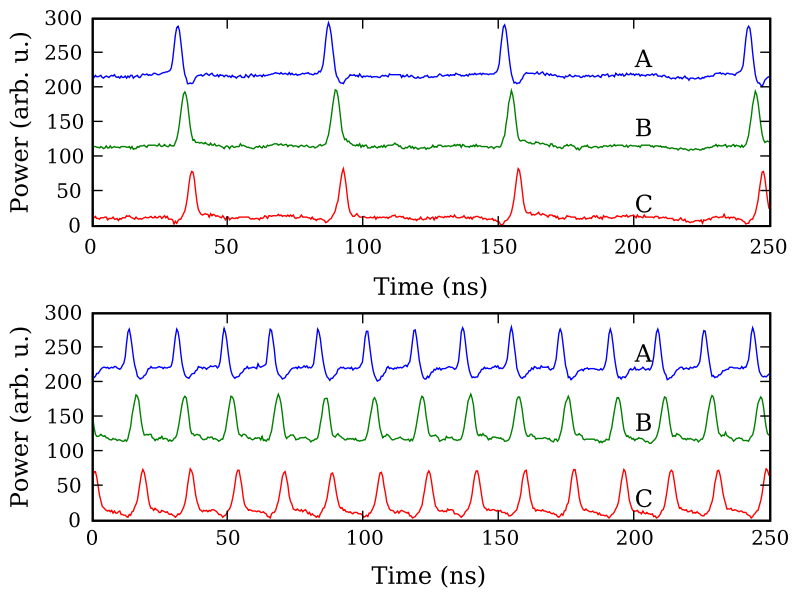

FIG. 2 (color online). Intensity outputs from a linear array of four detectors placed along a channel line and separated by $9 \mu \mathrm{m}$. Detector $A$ monitors the defect point where the structure forms, no intensity is monitored by the last detector of the array (detector $D$ ) and its trace is not shown. Each trace is vertically shifted for clarity. The VCSEL current is $441.76 \mathrm{~mA}$ (upper panel) and $442.0 \mathrm{~mA}$ (lower panel). HB intensity $=15 \mathrm{~mW}$. in the process. Further information can be obtained by Fig. 3, right panel, where we superimpose 50 realizations of pulse generation with the following evolutions monitored by the other two detectors. Even if we consider the situation where the process is characterized by the strongest dispersion, the traces recorded by the detectors $B$ and $C$ superimpose perfectly. This indicates clearly that the spatial evolution of the pulse is deterministic, being always the same after pulse formation in point $A$.

The results described above indicate the presence of a drifting CS that is originated from the structure appearing at point $A$, it immediately starts to drift along the channel due to the phase gradient with a speed of $3.0 \mu \mathrm{m} / \mathrm{ns}$, and eventually it dies out after detector $C$. Moreover, since at point $A$ the formation of the structure occurs regularly in time, a regular flow of moving CS settles down in the direction of the phase gradient. The formation time of the structure in point $A$, determines the period of the sequence, and it is dramatically affected by a variation of the pumping current of the device, while its drifting speed is not. The spontaneous formation of a stationary structure with high intensity in point $A$ once the phase gradient is removed, indicates that this point is a defect of the device section, i.e., a point where some system parameter value is locally different from the rest of the device. It is important to point out that, if the channel line is changed in the experiment (by changing the gradient direction and moving the detector array accordingly), other defect points of the device originating a regular flow of CS can be revealed. Their positions are randomly distributed throughout the transverse section of the device. The drift of the CS along the channel is interrupted after detector $C$ by the interaction with another defect which, in this case, annihilates the structure. The distance covered by the drifting CS before vanishing depends on the defect-free length of the channeling path. We observed drifting lengths spanning from 10 to $30 \mu \mathrm{m}$.

2D numerical simulations were performed in order to explain the above described spatiotemporal dynamics and
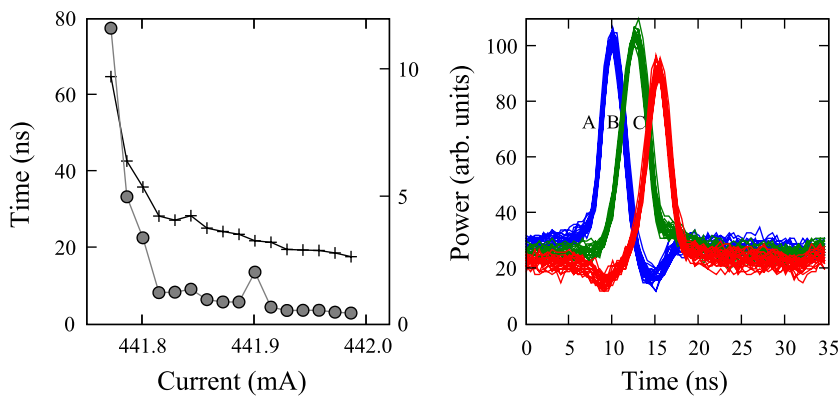

FIG. 3 (color online). Left Panel: $\langle T\rangle$ (crosses, with left vertical scale) and $\sigma(T)$ (circles, with right vertical scale) as a function of $J$. Right Panel: For the case depicted in Fig. 2, $J=$ $441.76 \mathrm{~mA}$, we superimpose 50 spatiotemporal sequences monitored by three detectors [from left to right: $A$ (blue curve), $B$ (green curve), $C$ (red curve)] along the channel. 
to confirm the role of device defects as sources of traveling CS. Furthermore, they allow us to understand the dependence of the phenomenon on the control parameters. We consider a rate equation model describing a driven VCSEL, pumped above transparency but below threshold. The dynamical equations, in the paraxial and mean field limit approximations, read [24]:

$$
\begin{gathered}
\frac{\partial E}{\partial t}=-\kappa[(1+i \theta) E-2 C(1-i \alpha) \\
\left.\times(N-1) E-E_{I}-i a \nabla_{\perp}^{2} E\right], \\
\frac{\partial N}{\partial t}=-\gamma\left[N+(N-1)|E|^{2}-I-d \nabla_{\perp}^{2} N\right],
\end{gathered}
$$

where $E$ is the normalized slowly varying envelope of the electric field and $N$ is the carrier density, $\kappa$ is the cavity decay rate, $\gamma$ is the nonradiative recombination rate, $\theta$ is the cavity detuning parameter $\theta=\left(\omega_{c}-\omega_{0}\right) / \kappa$, with $\omega_{0}$ being the injected frequency and $\omega_{c}$ the cavity frequency. $E_{I}$ is the normalized input field, $I$ is the normalized injected current, $2 C$ is the bistability parameter, $\alpha$ is the linewidth enhancement factor, and $\nabla_{\perp}^{2}$ is the transverse Laplacian where $a$ and $d$ are the diffraction and diffusion coefficients, respectively. The finite size of the device has been simulated by assuming an injected current with circular profile. A more detailed discussion of the parameter definitions and of the scalings can be found in [24]. The $S$-shaped input-output curve for the homogenous stationary solution of Eqs. (1) and (2), showing the intracavity field amplitude as a function of $\theta$, is shown in Fig. 4. The broken part of the curve shows the unstable region where, due to a modulational instability, the system generates a spatially modulated solution. CSs coexist with the stable low-intensity homogeneous solution for $-2.06 \leq \theta \leq$ -1.95 (that is, between the two dashed lines $a$ and $b$ ), while for $\theta<-2.06$ only patterns exist.

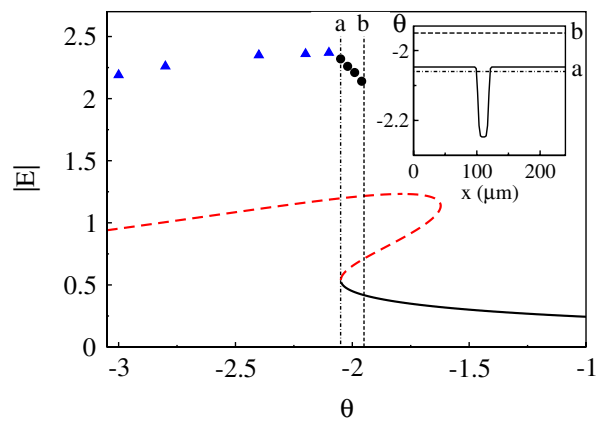

FIG. 4 (color online). Stationary solutions. The solid (dashed) line represents the stable (unstable) homogeneous steady state. Circles (triangles) represent the maximum intensity of stable CSs (patterns). Other parameters are, $I=2.0024, \alpha=5, d=$ $0.052, C=0.45, K=0.04 \mu \mathrm{m}^{-1}$ and $E_{I}=0.792$. Inset panel: Defect profile ( $x$ section): defect depth is $\Delta \theta=-0.2$ with respect the value of $\theta$ outside the defect $\theta=-2.0472$. The region between the two lines $a, b$ is the stability region of CSs.
Device defects in the transverse plane of the device are simulated by introducing a local spatial dependence in the value of the parameter $\theta$. In physical terms this means to identify the defects as local variations of the resonator thickness (due to Bragg reflectors roughness) [18,19]. This approach has been successfully used in order to reproduce numerically the device defect map experimentally observed [20]. A single defect is simply described by introducing the spatial profile of $\theta$ shown in the inset of Fig. 4. CSs are stable everywhere except in the defect where $\theta$ value is such that only the pattern solution is stable $(\theta<-2.06)$. According to the experimental observations, the defect size is assumed comparable with the CS size: the defect shape is assumed super-Gaussian with a diameter at half maximum of about $18 \mu \mathrm{m}$ ). A structure spontaneously forms upon this defect. This single peaked structure can be intuitively viewed as a "portion" of pattern, the characteristics of which are strongly dependent on the defect size and shape. At the same time, the gradient of $\theta(x, y)$ at the defect walls generates a pinning force on the structure. Finally we introduce a linear phase gradient $K$ in the holding beam, $E_{I}=E_{I 0} \exp (i \vec{K} \cdot \vec{x})$, with $K$ being strong enough to unpin the structure from the defect. This phase gradient pulls the structure immediately out from the defect, and bring it where both the CS and the low-intensity homogeneous solution are stable. Here the structure becomes a CS and it keeps on traveling in the direction of the gradient with a speed proportional to $K$ [13].

Numerical simulation of the spatiotemporal evolution of CS is represented in Fig. 5(a). The separation between two pulses is mainly due to the time required to regenerate the structure at the defect $(T)$ once the preceding one has gone.
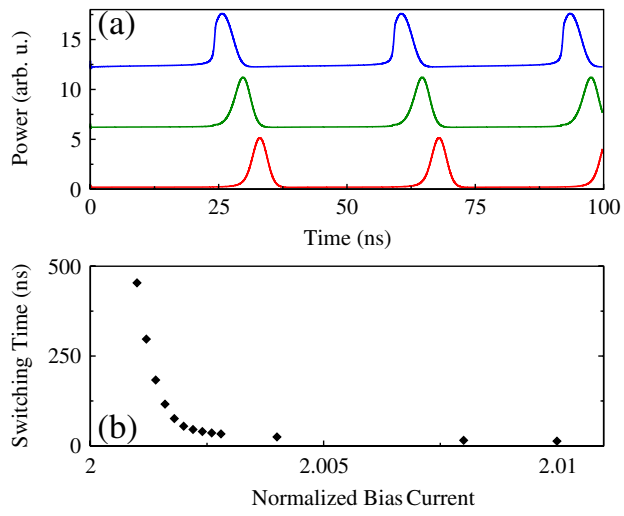

FIG. 5 (color online). (a) Numerically calculated intensity outputs from a (virtual) linear array of three detectors placed along the gradient direction. The upper trace corresponds to the output from a detector placed on the defect point. The middle (lower) trace corresponds to the output from a detector placed $9 \mu \mathrm{m}$ (18 $\mu \mathrm{m}$ ) from the defect. (b) Switching time of the structure $(T)$ as a function of $I$ keeping into account the dependence of $\theta$ on $I$ as observed experimentally. All parameters and defect profile as in Fig. 4. 
The dependence of the structure formation time on parameters can be understood as follows: once the structure has left the defect, the system relaxes back to the stable stationary state and the duration of this process depends critically on the parameter values inside the defect. In particular, it depends on how far these values are from the critical ones at which the homogeneous solution loses its stability. The divergence of $T$, as the control parameter approaches the bifurcation point, is a well-known behavior of systems close to bifurcation (critical slowing down) [25]. We have fit the dependence of the numerically obtained $T$ as a function of different control parameters $\left(I, \theta\right.$ and $\left.E_{I}\right)$ and we have found that it scales with the inverse of a square root law of $\left|\gamma-\gamma_{c}\right|$, with $\gamma$ being the generic parameter and $\gamma_{c}$ its critical value at the bifurcation, as predicted for critical slowing down in bistable system [25]. In order to reproduce numerically the dependence of $T$ on pumping current $(J)$ observed in the experiment, we have to take into account that an experimental variation of $J$ implies both a change of $I$ and a change of $\theta$ [26]. This is due to the change of the semiconductor refractive index caused by Joule heating. The dependence of $\theta$ on $I$ is phenomenologically introduced in the model by putting $\theta=\theta_{0}-5\left(I-I_{0}\right)$, being $\theta_{0}=-2.2472$ and $I_{0}=$ 2.0024. In Fig. 5(b) we plot the calculated values of $T$ as a function of $I$, once implemented this dependence. As in the experiment, $T$ diverges as $J$ approaches a critical value. Moreover, in the vicinity of the bifurcation, the noise is much more effective explaining why the fluctuations on $\langle T\rangle$ in Fig. 2 are larger for low $J . J$ cannot be increased arbitrarily since eventually the homogeneous solution becomes unstable even outside the defect and CSs do not exist anymore. This explains why value of $\langle T\rangle$ cannot be further reduced in Fig. 3, left panel. Defect depth and shape affects the characteristics of the reported spatiotemporal dynamics for CS but the onset of the periodic flow of drifting CS is rather robust versus defect characteristics. Three ingredients are necessary: outside the defect CS solution must be stable, inside the defect only pattern solution is stable and the phase gradient must be strong enough to push the structure outside the defect. The detailed analysis on the influence of defect characteristics on the spatiotemporal dynamics will be reported in a more extensive paper.

In conclusion, we have evidenced experimentally and analyzed numerically the CS spatiotemporal dynamics resulting from the interplay between a local defect and a phase gradient in the holding beam. This dynamics can be useful in order to engineer CS source points built in the device thus avoiding the use of a writing beam. Acting on the gradient strength and on system parameters, or by tailoring the defect depth, the characteristics of the CS periodic flow can be set. This paves the way to interesting solutions in order to decrease the complexity of the CS experimental apparatus: local perturbations of the resonator characteristics (reflectivity and thickness, for example) can be engineered in the transverse section of a device during the growth process. Moreover, the behavior upon variations of $J$ suggests that, by means of a weak modulation of the pumping current, it is possible to vary the period of the CS flow and even to stop the CS generation without perturbing the CS stability nor their speed outside the defect. This can be used as an optoelectronic method to store information bits in the form of moving CS.

This research was performed in the framework of the FET Open Project FunFACS. We thank Lionel Gil for useful discussions. G. Tissoni acknowledges the financial support of the Université de Nice Sophia-Antipolis.

[1] B. A. Malomed, Physica (Amsterdam) 29D, 155 (1987).

[2] O. Thual and S. Fauve, J. Math. Phys. (N.Y.) 49, 1829 (1988).

[3] Yu. Astrov and H. Purwins, Phys. Lett. A 283, 349 (2001).

[4] K. L. Lee et al., Nature (London) 369, 215 (1994).

[5] J. Wu et al., Phys. Rev. Lett. 52, 1421 (1984).

[6] E. Moses et al., Phys. Rev. A 35, 2757 (1987).

[7] M. Saffman et al., Opt. Lett. 19, 518 (1994).

[8] A. Schreiber et al., Opt. Commun. 136, 415 (1997).

[9] B. Schäpers et al., Phys. Rev. Lett. 85, 748 (2000).

[10] L. A. Lugiato, IEEE J. Quantum Electron. 39, 193 (2003).

[11] M. Tlidi et al., Phys. Rev. Lett. 73, 640 (1994).

[12] N. Rosanov and G. Khodova, Opt. Spectrosc. 65, 449 (1988).

[13] W. J. Firth and A. J. Scroggie, Phys. Rev. Lett. 76, 1623 (1996).

[14] M. Brambilla et al., Phys. Rev. Lett. 79, 2042 (1997).

[15] S. Barland et al., Nature (London) 419, 699 (2002).

[16] F. Pedaci et al., Appl. Phys. Lett. 89, 221111 (2006).

[17] F. Pedaci et al., Appl. Phys. Lett. 92, 011101 (2008).

[18] J. Oudar et al., Opt. Quantum Electron. 24, S193 (1992).

[19] R. Kuszelewicz et al., Phys. Rev. Lett. 84, 6006 (2000).

[20] F. Pedaci et al., Appl. Phys. Lett. 93, 111104 (2008).

[21] Periodic spatiotemporal dynamics of LS has been briefly mentioned in [7] though it was not explained nor further addressed. Preliminary results indicate that the same behavior is also present in liquid crystal light valve experiment [U. Bortolozzo, Institut Non Linéaire de Nice (private communication)].

[22] G. Liger-Belair et al., Phys. Rev. E 72, 037204 (2005).

[23] X. Hachair et al., Phys. Rev. A 69, 043817 (2004).

[24] L. Spinelli et al., Phys. Rev. A 58, 2542 (1998).

[25] F. Mitschke et al., Opt. Commun. 71, 385 (1989).

[26] X. Hachair et al., Phys. Rev. A 72, 013815 (2005). 